University of Wollongong

Research Online

Australian Institute for Innovative Materials -

Papers

Australian Institute for Innovative Materials

2013

Comparative displacement study of bilayer actuators comprising of conducting polymers, fabricated from polypyrrole, poly $(3,4-$ ethylenedioxythiophene) or poly(3,4-propylenedioxythiophene)

\author{
Babita Gaihre \\ University of Wollongong, babita@uow.edu.au \\ Syed Ashraf \\ University of Wollongong, syed@uow.edu.au \\ Geoffrey M. Spinks \\ University of Wollongong, gspinks@uow.edu.au \\ Peter C. Innis \\ University of Wollongong, peter_innis@uow.edu.au \\ Gordon G. Wallace \\ University of Wollongong, gwallace@uow.edu.au
}

Follow this and additional works at: https://ro.uow.edu.au/aiimpapers

Part of the Engineering Commons, and the Physical Sciences and Mathematics Commons

Research Online is the open access institutional repository for the University of Wollongong. For further information contact the UOW Library: research-pubs@uow.edu.au 


\title{
Comparative displacement study of bilayer actuators comprising of conducting polymers, fabricated from polypyrrole, poly(3,4- ethylenedioxythiophene) or poly(3,4-propylenedioxythiophene)
}

\author{
Abstract \\ The stress gradient generated due to electrochemical oxidation/reduction of a conducting polymer in a \\ bilayer actuator causes a bending movement. Here we report a comparative study of the displacement of \\ bilayer actuators prepared using different conducting polymers. The effects of conducting polymers \\ obtained from electrochemical or chemical synthesis route: different polymers (polypyrrole (PPy), \\ poly(3,4-ethylenedioxythiophene) PEDOT and poly(3,3-dihexyl-3-4-dihydro-2H-thieno[3,4b][1,4]-dioxepine \\ [PProDOT $(\mathrm{Hx}) 2$ ]); and different electrolytes (0.1 M lithium trifluromethanesulfonate/propylenecarbonate \\ (LiTFSI/PC) and 1-ethyl-3-methyl imidazolium trifluromethanesulfonate (EMITFSI)) on the displacement \\ amplitude and displacement patterns of these actuators were investigated and compared. The results \\ indicate that the bilayer actuator consisting of an electrochemically synthesized conducting polymer \\ exhibits far greater displacements compared to chemically synthesized polymers at the same stimulating \\ voltage. Similarly, amongst the three conducting polymers considered, the PPY actuator showed greater \\ displacement followed by PPrODOT and PEDOT. Furthermore, the use of different electrolyte solution \\ during polymerization as well as during actuation was found to affect not only displacement distance but \\ also its pattern.

\section{Keywords} \\ propylenedioxythiophene, comparative, study, displacement, bilayer, actuators, comprising, conducting, \\ polymers, fabricated, polypyrrole, poly, 3, 4, ethylenedioxythiophene \\ Disciplines \\ Engineering | Physical Sciences and Mathematics

\section{Publication Details} \\ Gaihre, B., Ashraf, S., Spinks, G. M., Innis, P. C. \& Wallace, G. G. (2013). Comparative displacement study of \\ bilayer actuators comprising of conducting polymers, fabricated from polypyrrole, poly $(3,4-$ \\ ethylenedioxythiophene) or poly(3,4-propylenedioxythiophene). Sensors and Actuators, A: Physical, 193 \\ (April), 48-53.
}




\section{Comparative displacement study of bilayer actuators comprising of conducting polymers, fabricated from polypyrrole, poly (3,4-ethylenedioxythiophene) or poly (3,4- propylenedioxythiophene)}

Babita Gaihre, Syed Ashraf, Geoffrey M. Spinks, Peter C. Innis, Gordon G. Wallace ARC Centre Excellence for Electromaterials Science, University of Wollongong, Intelligent Polymer Research Institute, Innovation Campus, NSW 2522, Australia

\section{$\underline{\text { Abstract }}$}

The stress gradient generated due to electrochemical oxidation/reduction of a conducting polymer in a bilayer actuator causes a bending movement. Here we report a comparative study of the displacement of bilayer actuators prepared using different conducting polymers. The effects of conducting polymers obtained from electrochemical or chemical synthesis route; different polymers ((polypyrrole (PPy), poly (3,4-ethylenedioxythiophene) PEDOT and poly (3,3-dihexyl-3-4-dihydro2H-thieno[3,4b][1,4]-dioxepine [PProDOT(Hx) $\left.)_{2}\right)$; and different electrolytes (0.1 M Lithium trifluromethanesulfonate/propylenecarbonate (LiTFSI/PC) and 1-ethyl-3-methyl imidazolium trifluromethanesulfonate (EMITFSI) on the displacement amplitude and displacement patterns of these actuators were investigated and compared. The results indicate that the bilayer actuator consisting of an electrochemically synthesized conducting polymer exhibits far greater displacements compared to chemically synthesized polymers at the same stimulating voltage. Similarly, amongst the three conducting polymers considered, the PPY actuator showed greater displacement followed by PProDOT and PEDOT. Furthermore, the use of different electrolyte solution during polymerization as well as during actuation was found to affect not only displacement distance but also its pattern.

Keywords: bilayer actuators; conducting polymers; displacement; polypyrrole; poly (3,4ethylenedioxythiophene); poly (3,4-propylenedioxythiophene); ionic liquid

\section{Introduction}

Conducting polymers exhibit electrochemically driven expansion and shrinkage due to ingress and egress of dopant ions in response to the electrochemical potential applied. The contraction and expansion of the conducting polymer can be converted to a reversible bending motion if a second layer, which does not change volume upon exposure to the electrochemical stimuli, is laminated to the conducting polymer film. During the electrochemical oxidation/reduction process the inert and flexible layer generates a stress gradient at the polymer interface due to the expansion/contraction of the conducting polymer film. This stress gradient causes a bending movement away from or toward the side of the conducting polymer film depending on the voltage applied. Most of these bending 
type actuators have been constructed based on either bilayer [1] or trilayer configuration [2, 3] to achieve a bending movement. Adhesive tapes [4], polymer films [2, 3] and thin deposited metal layers [1] have been the materials most widely used as the inert, flexible substrate in bilayer and trilayer actuators. The trilayer actuators are capable of working in both air and liquid since the middle layer acts as the electrolyte. Bilayers are capable of working in a liquid electrolyte medium only.

Several problems remain in the practical application of these conducting polymer bending actuators. Delamination initiated by repeated volume change at the interface causes irreversible deterioration of actuator performance. To resolve the problem of delamination, Vidal et al. proposed a new actuator concept, that utilised an interpenetrating polymer network (IPN) consisting of embedded conducting polymer [5, 6]. Khaldi et al. demonstrated fabrication of microactuators made up of such IPN [7]. Sansinena et al. also reported monolithic type polyaniline electrochemical actuators [8] where the density of the film changed throughout the film thickness and resulted in a bending action. Wu et al. have also described the use of a porous membrane coated first in metal and then with conducting polymer so that the rough membrane surface provided adequate resistance to delamination [9].

A second problem of solvent evaporation for air-operated bending actuators can be resolved by the use of ionic liquids. These liquids are organic/inorganic salts with good chemical and thermal stability, non-volatile and non-flammable and show high ionic conductivity. Cho et al. introduced a single layer actuation system based on PEDOT and nitrile rubber (NBR) containing room temperature ionic liquid as a solid polymer electrolyte [10-12]. The actuator performance was found to be dependent on anion-size of the ionic liquid as well as amount of acrylonitrile in the NBR.

In this paper, we report on comparative displacement study of the bilayer actuator based on three different conducting polymers that have been synthesized in different conditions. Firstly, the effect of electrochemical synthesis and chemical synthesis method on the displacement of the bilayer actuator was investigated. Researchers have used two routes viz. chemical [5-8, 10-12] and electrochemical [1-3] synthesis routes for the preparation of organic polymer actuators. While most of the PPY actuators have been synthesized by electrochemical routes [1-3], most of the PEDOT actuators have been synthesized by chemical routes [5-8, 10-12]. To date there has been no systematic study of the effect of synthesis method on the actuation performance.

The second parameter studied was the type of conducting polymer. A comparison was made of bilayer actuators fabricated from PPy, PEDOT and PProDOT. While most researchers have focused on synthesis and characterization of the actuators based on PEDOT and PPy, none has 
considered comparing the performance of the actuator synthesized from these conducting polymers. Similarly, several researchers have focused on electrochromic [14], photovoltaic [15] and supercapacitor [16] applications of the PProDOT, but no work has been dedicated towards synthesizing and characterization of actuation behaviour of this polymer.

The third parameter studied was the type of electrolyte. A conventional organic electrolyte (0.1 M LiTFSI/PC) was compared with an ionic liquid (1-ethyl-3-methyl imidazolium bis (trifluoromethylsulfonyl) imide EMITFSI.

\section{Experimental}

\subsection{Chemicals and Materials}

Polyvinylidene (PVDF) membrane with thickness 110 microns was purchased from Millipore. Pyrrole (Merck) was distilled and stored under nitrogen at $-20{ }^{0} \mathrm{C}$. 3,4-ethylenedioxythiophene was purchased from H.C. Starck. PEDOT-PSS (Orgacon dry) was purchased from Agfa.

\subsection{Synthesis of $\operatorname{ProDOT}(\mathrm{Hx})_{2}$ monomer}

Synthesis of [ProDOT(Hx) $)_{2}$ ] was carried out, following the literature [17] method with some modification. First 2,2-dihexyl-propane-1,3-diol was synthesized by an alkylation of diethyl malonate using sodium hydride and the corresponding alkyl bromide followed by $\mathrm{LiAlH}_{4}$ reduction.

Transetherification of 3,4-dimethoxy thiophene with above diol resulted in the corresponding 3,3-dihexyl-3,4-dihydro-2H-thieno[3,4-b][1,4]-dioxypine [ProDOT(Hx) $\left.)_{2}\right]$ after twice purification by column chromatography. Bromination of [ProDOT(Hx) $)_{2}$ resulted 6,8-dibromo-3,3-Dihexyl-3,4dihydro-2H-thieno[3,4-b][1,4]-dioxypine[ProDOT( $\left.\left(\mathrm{Hx}_{2}\right)_{2} \mathrm{Br}_{2}\right]$ and purified by column chromatography.

\subsection{Chemical synthesis of PProDOT $(\mathrm{Hx})_{2}$ polymer}

Chemical polymerization of ProDOT(HX) $)_{2} \mathrm{Br}_{2}$ was carried out by using Grignard's reagent (methyl magnesium bromide $1 \mathrm{M}$ in $\mathrm{THF}$ ) in presence of $\mathrm{Ni}(\mathrm{dppp}) \mathrm{Cl}_{2}$ catalyst followed by purification using soxhlet extraction with methanol 24h, hexane $48 \mathrm{~h}$ and chloroform $24 \mathrm{~h}$. After every step of the reaction the product was characterized by NMR which was in full agreement with the described structure. The polymer was also characterized by gel permeation chromatography (GPC) method using polystyrene as standard (Mn 5363; Mw 15500; PDI 2.8).

\subsection{Synthesis of [EMI-TFSI]: Modified (literature method [18])}

Under dry and inert condition, 147gm bromoethane was added drop wise to the stirred 37gm 1methylimidazole. The reaction mixture was stirred for $48 \mathrm{~h}$ (the white solid formation was broken 
down by spatula at various intervals). The solid was washed twice with diethyl ether (decanted) and dried on rotary evaporator. This dry white solid (hygroscopic) 1-ethyl-3-methylimidazolium bromide ( $\mathrm{EMI}^{+} \mathrm{Br}^{-}$) was kept in desiccator until further use for the next reaction step.

$50 \mathrm{gm}\left(\mathrm{EMI}^{+} \mathrm{Br}^{-}\right)$was dissolved in $200 \mathrm{~mL}$ water and mixed with equal molar of lithium trifluorosulfonylamide dissolved in $200 \mathrm{~mL}$ water, stirred for $2 \mathrm{~h}$. The phase separated ionic liquid was washed ( 2 x 200ml water) and bubbled with $\mathrm{N}_{2}$ at $90{ }^{0} \mathrm{C}$ for a few hours.

\subsection{Gold sputter coating}

Gold was sputter coated using Magnetron sputter coater SC 100MS on one side of the PVDF membrane at $2 \times 10^{-3} \mathrm{mBar}$ pressure and $30 \mathrm{~mA}$ current. The coated layers of gold served to provide a conductive surface onto which the conducting layers could be electrodeposited. The coating time was fixed at $20 \mathrm{~min}$.

\subsection{Bilayer actuator preparation using spray coating and electrochemical coating technique Bilayer actuator}

Figure 1a shows a schematic diagram of the bilayer actuator. Bilayer actuators consisting of chemically synthesized conducting polymers (PEDOT and PProDOT(HX) $)_{2}$ ) were prepared by spray coating technique. Aqueous solution of the conducting polymer was spray coated on the gold coated PVDF membrane and the membrane was left at $50^{\circ} \mathrm{C}$ for drying. As PPy is insoluble in aqueous solution, we have not used this polymer to obtain chemically synthesized PPy actuator.

Similarly, bilayer actuators consisting of electrochemically synthesized conducting polymers (PPy, PEDOT and PProDOT $(\mathrm{HX})_{2}$ ) were prepared by galvanostatic polymerization technique. Gold coated PVDF membrane was used as the working electrode and stainless steel mesh was used as the counter electrode. A potentiostat/galvanostat (EG\&G Princeton Applied Research Model 363) was used to generate constant current into the polymerization solution. Monomer concentration and current density were $0.1 \mathrm{M}$ and $0.1 \mathrm{mAcm}^{-2}$ and the polymerization solutions were degassed before polymerization. Two different types of electrolyte solution, $0.1 \mathrm{M} \mathrm{LiTFSI/PC} \mathrm{(with} \mathrm{1 \%} \mathrm{water)} \mathrm{and}$ EMITFSI and three different types of conducting polymers, PPy, PEDOT and PProDOT(HX $)_{2}$ were chosen for comparative study. PPy was polymerized at $-32^{0} \mathrm{C}$ but PEDOT and ProDOT(HX $)_{2}$ were polymerized at room temperature as lower temperature gave poor quality deposits.

\subsection{Electromechanical response of the actuators}

Due to the mechanical constraint imposed by the PVDF layer, the bilayer bends to one direction depending on the voltage applied and doping ion used (Figure 1b). The electromechanical 
response of the actuators under different voltage and frequencies were observed in two different types of electrolyte solutions, 0.1M LiTFSI/PC and EMITFSI. A platinum mesh was used as counter electrode and $\mathrm{Ag} / \mathrm{Ag}^{+}$was used as reference electrode.

E-corder, (ED821) was an interface unit between the computer and the potentiostat, which recorded a voltage signal applied to the bilayer actuator and associated current drawn. The actuator input voltage signals were provided by an external functional generator and amplified using an eDAQ potentiostat (EA161) operating in a three-electrode mode. The displacement distance of the bilayer actuators were measured using paper scale glued to the wall of the cell from exterior.

\section{Results and Discussion \\ Displacement Amplitude}

Displacement of bilayer actuators comprising of chemically synthesized PEDOT-PSS and PProDOT $(\mathrm{Hx})_{2}$ was compared with that of electrochemically synthesized polymers. The chemically synthesized conducting polymers were coated on the gold coated PVDF membrane using spray coating technique. It was found that displacement of electrochemically synthesized actuators showed far better displacement than the chemically synthesized ones (Figure 2) when the bilayers were stimulated using a square wave potential between $0 \mathrm{~V}$ and $+0.05 \mathrm{~V}$ at $1 \mathrm{mHz}$. Thickness of conducting polymers in both cases was kept same. Electropolymerization technique has been found to results in the conducting polymer films that are highly conductive than the ones, which are polymerized by chemical polymerization technique. And as discussed earlier shrinkage and expansion of the conducting polymer is a function of amount of ions ingress and egress from it. The more ions enter in or out of the conducting polymer more shrinkage or expansion of the conducting polymer occurs, which result in greater displacement of the conducting polymers. Since electrochemically synthesized conducting polymers are more conducting, comparably more ions enter in or out of the polymer, resulting in more displacement. This is the reason for the better performance of the electrochemically synthesized actuators.

On comparison of electrochemically synthesized PEDOT, PProDOT(Hx $)_{2}$ and PPy, it was found that PPy showed displacement which was around 5 times greater than PEDOT and 2 times greater than PProDOT $(\mathrm{Hx})_{2}$ at 0.5 volts (Figure 2 and 3a).

Voltage dependent displacement was also measured for all the three types of electrochemically synthesised actuators in 0.1 M LiTFSI/ PC (Figure 3a) and EMITFSI (Figure 3b). These actuators were all synthesized in 0.1M LiTFSI/PC electrolyte. All the actuators showed an approximately linear increase in displacement with increasing voltage. However, the slope of 
lines for PPy, PProDOT(HX) $)_{2}$ and PEDOT, which are $~ 25,9$ and $7 \mathrm{~mm} / \mathrm{V}$, respectively suggests that the increase in displacement with increasing voltage is not uniform for each of these conducting polymers (Figure 3a). Furthermore, a decrease in displacement occurred when actuation was observed in EMITFSI (Figure 3b) compared to 0.1M LiTFSI/PC (Figure 3a). It must be noted that although thickness of PPy was smaller than PProDOT(HX) $)_{2}$ and PEDOT, the displacement shown by corresponding actuator was larger. The reason for this is still unknown and is subject of more extensive research.

The voltage dependent displacement was also measured for PPY, synthesized in EMITFSI and compared with the polymer synthesized in LiTFSI/PC. It was found that the PPy synthesized from EMITFSI gave a similar displacement as that of PPy synthesized in 0.1 M LiTFSI/PC (Figure 4a), especially at lower voltage. The displacement study was done in EMITFSI. However, the displacement of the PPy synthesized in EMITFSI was further reduced to half when LiTFSI/PC was used as electrolyte for the actuation study (Figure 4b). Although in both cases the dopant ion is the TFSI ${ }^{-}$ion, it appears that the electrolyte used during polymerization process plays a significant role in determining the actuation magnitude. If polymerization is done in EMITFSI then the actuator shows a larger displacement in EMITFSI but if the polymerization is done in LiTFSI/PC than actuator shows larger displacement in the LiTFSI/PC.

Frequency dependent displacements of the three different types of actuators were studied in ionic liquid (Figure 5) and in each case the amplitude of displacement decreased with increasing frequency. This behaviour is expected because the ion diffusion controls the rate of the electrochemical reactions and at higher frequencies, there is less time for the diffusion of ions in and out of the polymer [2]. As a result of the reduced ion flux, the charge transferred is also smaller at higher frequencies leading to a reduced bending motion.

The speed of actuation for PPy actuators, synthesized in 0.1M LiTFSI/PC, was also compared in the ionic liquid and LiTFSI/PC solution (Figure 6a). It was found that PPy actuator took less than ten seconds to travel $7 \mathrm{~mm}$ of distance in LiTFSI/PC solution, while in the ionic liquid the speed reduced and the actuator took around 25 second to travel $5 \mathrm{~mm}$ of distance and remaining 2 mm of displacement was achieved in 75 seconds (Figure $\mathbf{6 b}$ ). The speed of displacement was also recorded for PProDOT(HX) $)_{2}$ in EMITFSI (Figure 6b) and found to be much lower than PPy in the corresponding solution. 


\section{Displacement pattern study}

The displacement pattern of PPy grown in two different electrolytes, $0.1 \mathrm{M} \mathrm{LiTFSI/PC}$ and EMITFSI was investigated and compared when tested in two different types of electrolyte solutions, 0.1M LiTFSI/PC and EMITFSI. It was found that the PPy grown in the 0.1M LiTFSI/PC electrolyte solution showed expansions when a positive voltage was applied (Figure 7, left) and contraction when a negative voltage was applied (Figure 7, right). The electrolyte solution used for the actuation was $0.1 \mathrm{M}$ LiTFSI solution. These observations indicate that the TFSI- ion moves in and out of the PPy lead to expansion and contraction during oxidation and reduction, respectively.

However, the same actuator when actuated in EMITFSI solution showed a different behaviour. First the PPy layer contracted suddenly and then expanded slowly to the original position when a positive voltage was applied, as shown in Figure 8. This behaviour was not observed at $0.1 \mathrm{~Hz}$ and higher frequencies. At these higher speeds, the slow relaxation was not observed. Furthermore, PEDOT also showed the same behaviour as the PPy. However, this behaviour was not observed by PProDOT(HX) $)_{2}$. It expanded and contracted normally both in LiTFSI/PC and EMITFSI solution as shown in Figure 7.

A similar actuation study was also conducted on the bilayer actuator grown in EMITFSI. The actuation observed in 0.1M LiTFSI/PC. was normal as shown in Figure 7 with the TFSI anion moving in and out of the polymer during oxidation and reduction, respectively. Interestingly, the displacement pattern was exactly opposite when actuation was done in EMITFSI. That is, rather than showing expansion, it showed contraction under positive voltage and expansion under negative voltage. In later case, movement of $\mathrm{EMI}^{+}$is occurring rather than TFSI${ }^{-}$.

\section{Conclusions}

The study concludes that electrochemically synthesized conducting polymers will give far greater displacement compared to chemically synthesized ones. Amongst three different types of electrochemically synthesized conducting polymers, PPy generate great displacement followed by PProDOT $(\mathrm{HX})_{2}$ and PEDOT. Displacement pattern was found to be altered when EMITFSI was used as electrolyte solution. Not only, the displacement pattern but also the speed of actuation as well as amount of displacement was found to be dependent on the type of electrolyte used during polymerization as well as displacement measurement. 


\section{Acknowledgement}

Authors would like to thank Australian Research Council (ARC), ARC centre excellence for electromaterials science (ACES) and Australian National Fabrication Facility (ANFF) for financial support to conduct this research.

\section{References}

[1] E Smela, Conjugated Polymer Actuators for Biomedical Applications, Advanced Materials, 15(2003) 48194.

[2] B Gaihre, G Alici, GM Spinks, JM Cairney, Effect of electrolyte storage layer on performance of PPyPVDF-PPy microactuators, Sensors and Actuators B: Chemical, 155(2011) 810-6.

[3] B Gaihre, G Alici, GM Spinks, JM Cairney, Synthesis and performance evaluation of thin film PPy-PVDF multilayer electroactive polymer actuators, Sensors and Actuators A: Physical, 165(2011) 321-8.

[4] TF Otero, JM Sansieña, Soft and Wet Conducting Polymers for Artificial Muscles, Advanced Materials, 10(1998) 491-4.

[5] F Vidal, C Plesse, D Teyssié, C Chevrot, Long-life air working conducting semi-IPN/ionic liquid based actuator, Synthetic Metals, 142(2004) 287-91.

[6] F Vidal, C Plesse, G Palaprat, A Kheddar, J Citerin, D Teyssié, et al., Conducting IPN actuators: From polymer chemistry to actuator with linear actuation, Synthetic Metals, 156(2006) 1299-304.

[7] A Khaldi, C Plesse, C Soyer, E Cattan, F Vidal, C Legrand, et al., Conducting interpenetrating polymer network sized to fabricate microactuators, Appl Phys Lett, 98(2011).

[8] JM Sansiñena, J Gao, HL Wang, High-Performance, Monolithic Polyaniline Electrochemical Actuators, Advanced Functional Materials, 13(2003) 703-9.

[9] Y Wu, G Alici, GM Spinks, GG Wallace, Fast trilayer polypyrrole bending actuators for high speed applications, Synthetic Metals, 156(2006) 1017-22.

[10] M Cho, H Seo, J Nam, H Choi, J Koo, Y Lee, High ionic conductivity and mechanical strength of solid polymer electrolytes based on NBR/ionic liquid and its application to an electrochemical actuator, Sensors and Actuators B: Chemical, 128(2007) 70-4.

[11] MS Cho, JD Nam, Y Lee, HR Choi, JC Koo, Dry Type Conducting Polymer Actuator Based on PolypyrroleNBR/Ionic Liquid System, Molecular Crystals and Liquid Crystals, 444(2006) 241-6.

[12] MS Cho, HJ Seo, JD Nam, HR Choi, JC Koo, KG Song, et al., A solid state actuator based on the PEDOT/NBR system, Sensors and Actuators B: Chemical, 119(2006) 621-4.

[13] H Yamato, M Ohwa, W Wernet, Stability of polypyrrole and poly(3,4-ethylenedioxythiophene) for biosensor application, J Electroanal Chem, 397(1995) 163-70.

[14] G Gunbas, L Toppare, Electrochromic conjugated polyheterocycles and derivatives-highlights from the last decade towards realization of long lived aspirations, Chemical Communications, 48(2012) 1083-101.

[15] J-H Huang, Z-Y Ho, D Kekuda, C-W Chu, K-C Ho, Controlled Growth of Nanofiber Network Hole Collection Layers with Pore Structure for Polymer-Fullerene Solar Cells, The Journal of Physical Chemistry C, 112(2008) 19125-30.

[16] MR Rosario-Canales, P Deria, MJ Therien, JJ Santiago-Avilés, Composite Electronic Materials Based on Poly(3,4-propylenedioxythiophene) and Highly Charged Poly(aryleneethynylene)-Wrapped Carbon Nanotubes for Supercapacitors, ACS Applied Materials \& Interfaces, 4(2011) 102-9.

[17] BD Reeves, CRG Grenier, AA Argun, A Cirpan, TD McCarley, JR Reynolds, Spray Coatable Electrochromic Dioxythiophene Polymers with High Coloration Efficiencies, Macromolecules, 37(2004) 7559-69.

[18] P Bonhôte, A-P Dias, N Papageorgiou, K Kalyanasundaram, M Grätzel, Hydrophobic, Highly Conductive Ambient-Temperature Molten Saltst, Inorganic Chemistry, 35(1996) 1168-78. 


\section{Figure captions}

Figure 1 a) Schematic representation of bilayer actuator and b) working principle of the bending type actuator.

Figure 2. Displacement of bilayer actuators consisting of chemically and electrochemically synthesized conducting polymers.

Figure 3. Voltage dependent displacement of bilayer actuators consisting of electrochemically synthesized conducting polymers in 0.1M LiTFSI/PC (a) and EMITFSI (b).

Figure 4. Displacement of PPy actuators. Polymer grown in different electrolyte and actuated in EMITFSI (a). Polymers grown in same electrolyte and actuated in EMITFSI and 0.1M LiTFSI/PC(b).

Figure 5. Frequency dependent displacement of the actuators in ionic liquid. The actuators were synthesized in 0.1 MLiTFSI/PC.

Figure 6. Speed of displacement of PPy in EMITFSI and LiTFSI/PC (a) and PPy and PProDOT(HX) $)_{2}$ in EMITFSI.

Figure 7. Displacement of bilayer actuator when TFSI is used as dopant in LiTFSI/PC solution.

Figure 8. Displacement pattern of PPy and PEDOT in EMITFSI solution. 
Figures

(a)

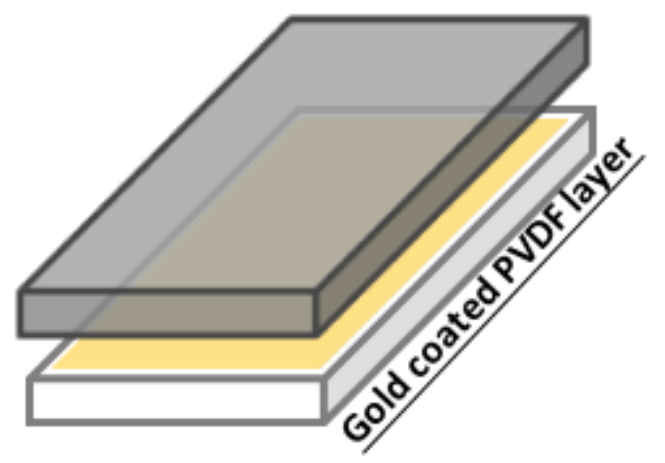

(b)

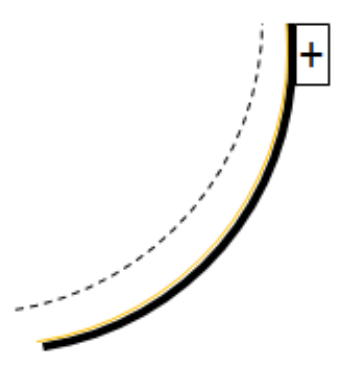

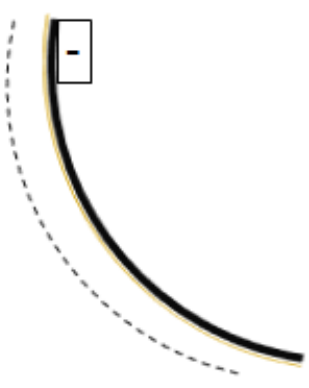

Figure 1 


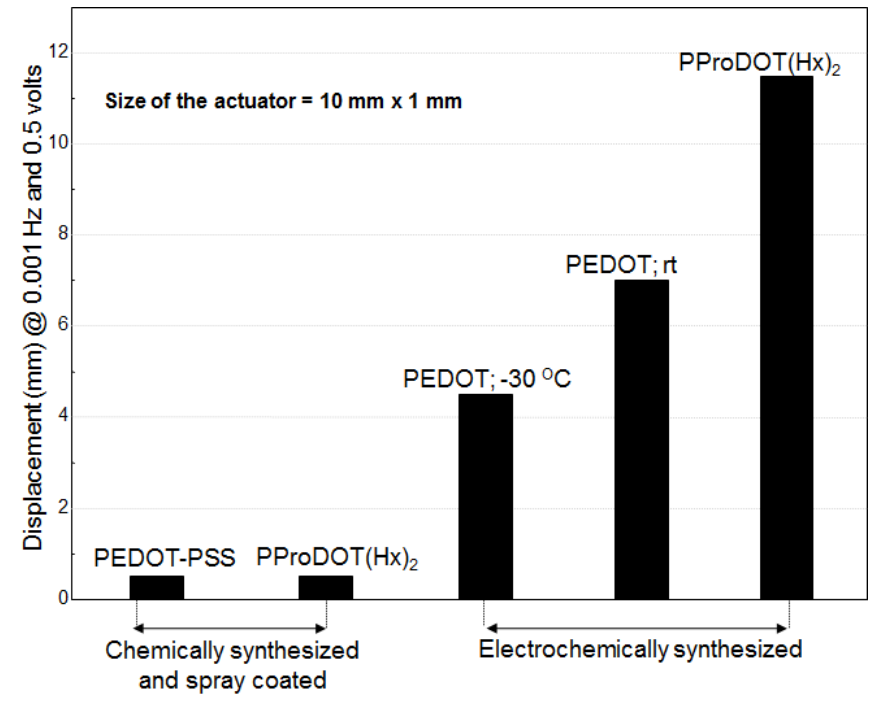

Figure 2 

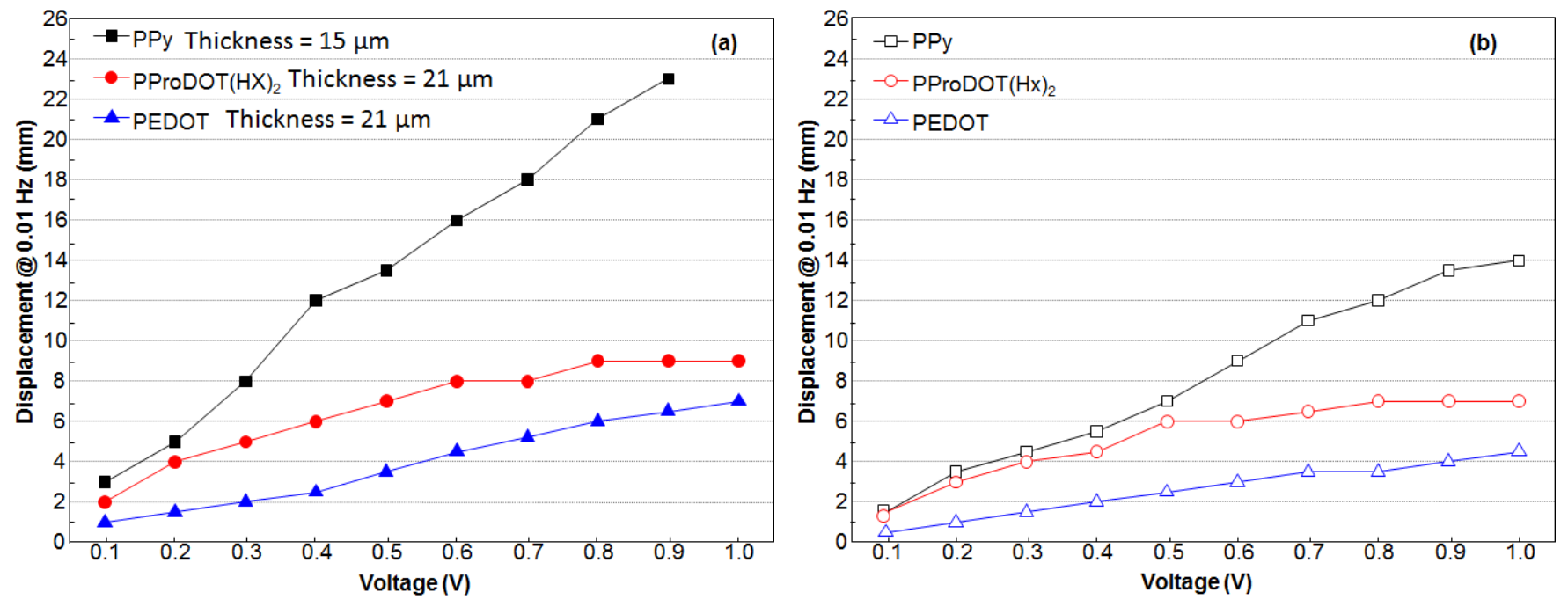

Figure 3 

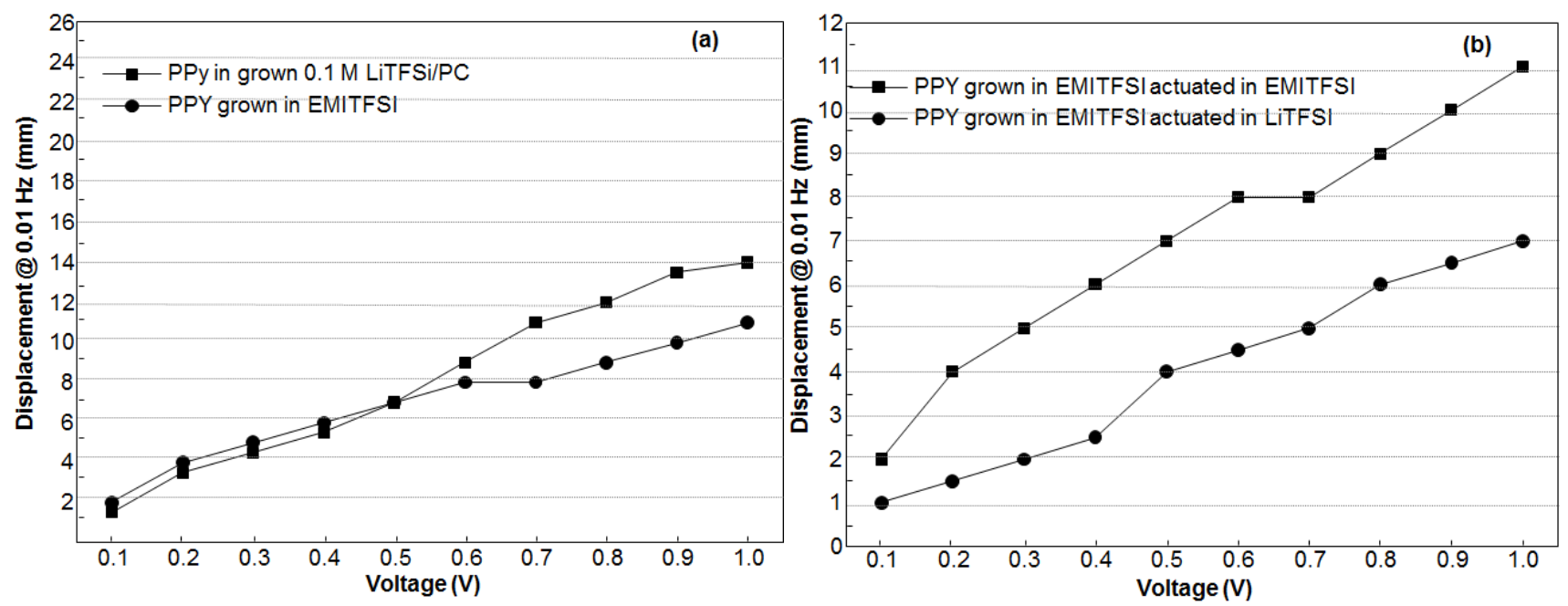

Figure 4 


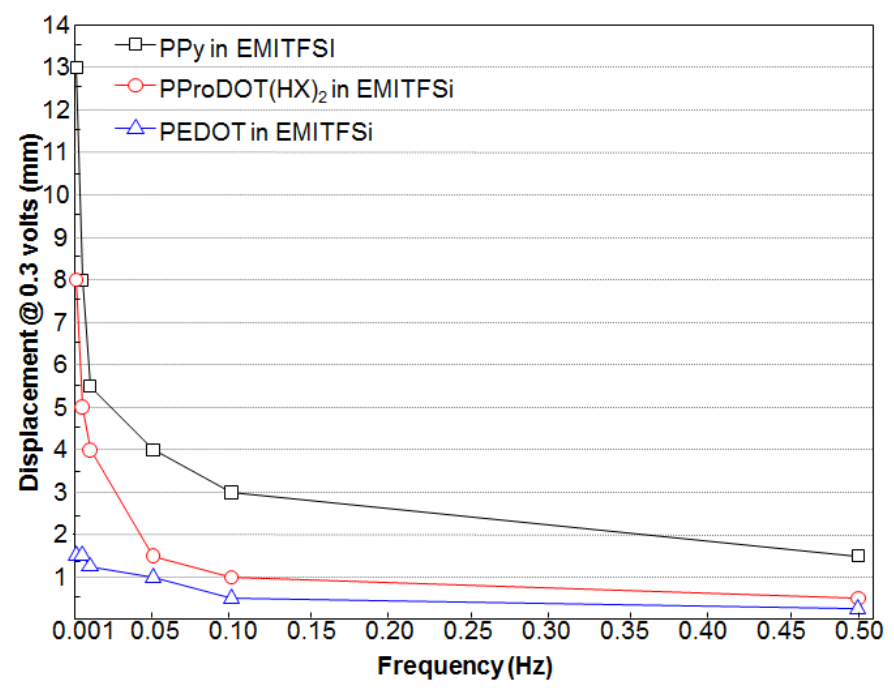

Figure 5 

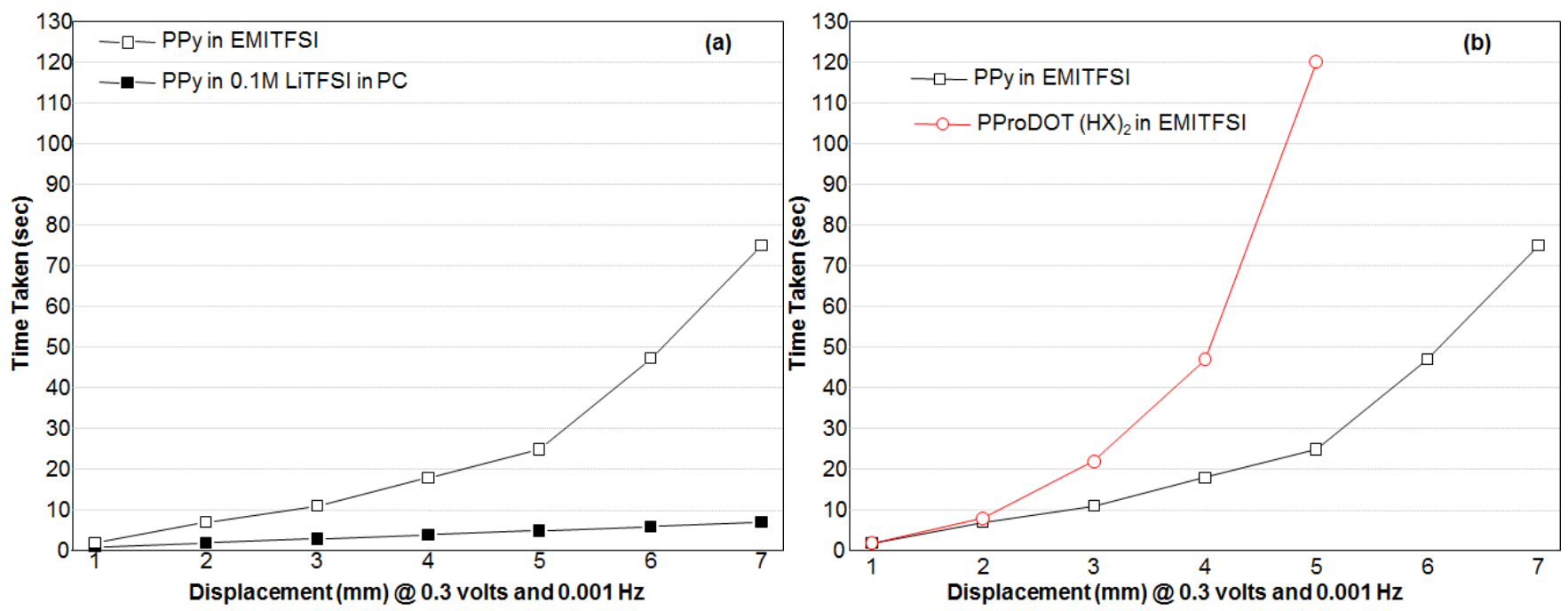

Figure 6 


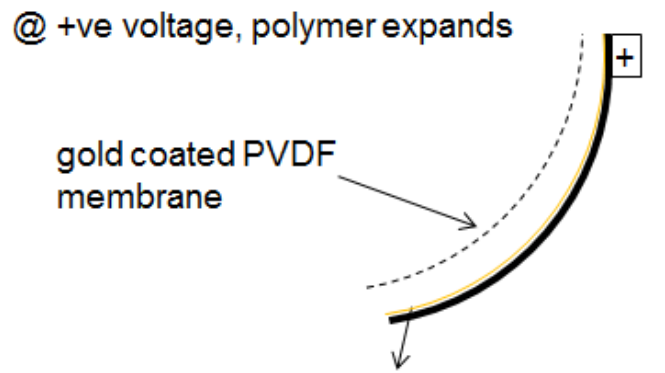

conducting polymer

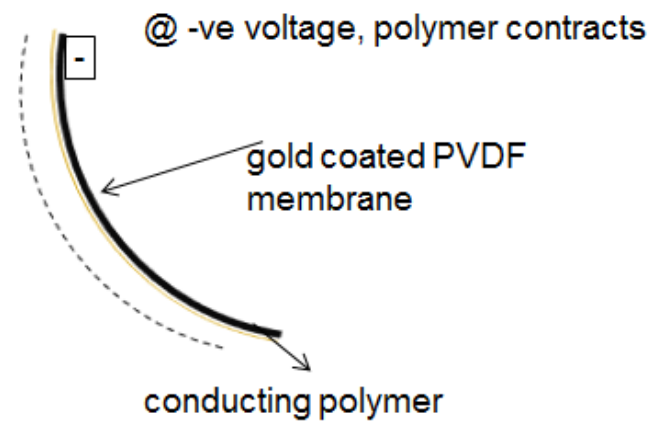

conducting polymer

Figure 7 


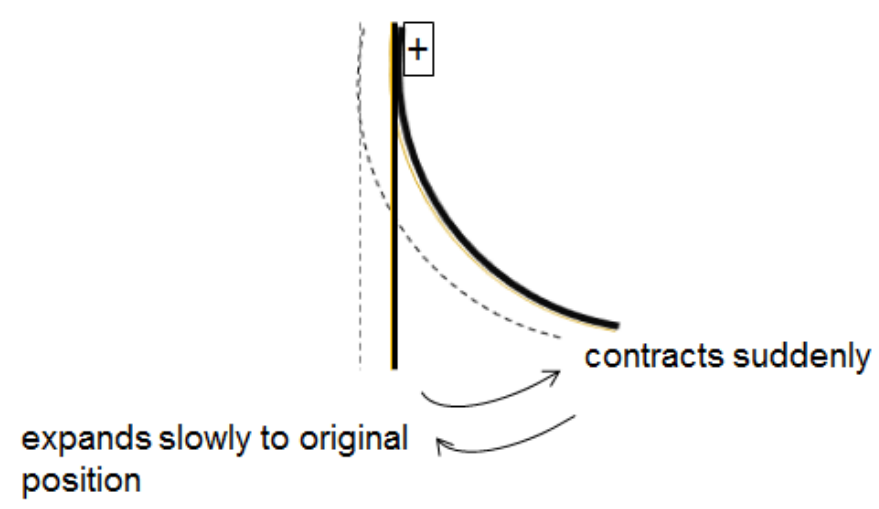

Figure 8 